\title{
Chromosome 5 and Parkinson disease
}

\author{
Tatiana Foroud ${ }^{* 1}$, Nathan Pankratz ${ }^{1}$, Maria Martinez ${ }^{2}$ and the PROGENI/GSPD-European \\ Consortium $^{3}$
}

\author{
${ }^{1}$ Indiana University School of Medicine, Indianapolis, IN, USA; ${ }^{2}$ INSERM EMIOO-06, 523 Place des Terrasses \\ de l'Agora, Evry, France
}

Parkinson disease (PD) is the second most common neurodegenerative disorder. Despite the identification of five causative genes, the majority of PD etiology is still unknown. A region on chromosome $5 q$ is one of the few regions of the genome found linked in multiple studies of familial PD. Analyses were performed using genotypic data from two independent research studies to evaluate rigorously the evidence of linkage on chromosome 5. The combined sample consisting of 1238 affected individuals from 569 multiplex PD families were genotyped for a common set of 20 microsatellite markers spanning an $80 \mathrm{cM}$ region on chromosome 5q. Two disease models were employed and model-free linkage analyses were performed to detect linkage to a PD susceptibility gene and also to detect linkage to a quantitative phenotype, age of onset of PD. There was little evidence of linkage using either a narrower or broader disease definition (lod $<0.5$ ). Analyses employing age of onset of PD as the phenotype produced a lod score of 1.8 . These results in a very large sample of familial PD suggest that it is unlikely that a PD susceptibility gene is located on chromosome 5q. Evidence for a locus contributing to the age of onset of PD is modest at best (empirical $P$-value $=0.07$ ).

European Journal of Human Genetics (2006) 14, 1106-1110. doi:10.1038/sj.ejhg.5201666; published online 31 May 2006

Keywords: Parkinson disease; chromosome 5; linkage analysis

\section{Introduction}

Parkinson disease (PD) is the second most common neurodegenerative disorder. There is wide variability in the age of onset of disease, although the average age is approximately 60 years. ${ }^{1} \mathrm{PD}$ is characterized by bradykinesia, resting tremor, muscular rigidity and postural instability, as well as a clinically significant response to treatment with levodopa. ${ }^{2}$ Recent studies have consistently found a significant genetic contribution to the risk of PD. Genetic analyses have identified five genes, which when mutated can result in PD. ${ }^{3}$ Two of these genes, SNCA and

\footnotetext{
*Correspondence: Dr T Foroud, Department of Medical and Molecular Genetics (IB 130), Indiana University School of Medicine, 975 West Walnut Street, Indianapolis, IN 46202-5251, USA. Tel: + 1317278 1291; Fax: + 1317278 1100;

E-mail: tforoud@iupui.edu

${ }^{3}$ For more details regarding PROGENI/GSPD-European Consortium see Appendix A.

Received 14 February 2006; revised 26 April 2006; accepted 27 April 2006; published online 31 May 2006
}

LRRK2, act in an autosomal dominant manner whereas the other three genes, PRKN, PINK1 and DJ1, are autosomal recessive in their mode of action. The majority of families with PD are not segregating a simple Mendelian form of disease. Rather, in many of these families, particularly those with multiple members with PD, it is expected that PD susceptibility genes may be interacting with each other or environmental factors, thereby increasing the risk of PD. Ongoing studies of multiplex PD pedigrees have provided evidence of linkage to several chromosomal regions.

Chromosome $5 q$ is one of the few regions of the genome identified as a locus for PD susceptibility in multiple studies (Figure 1). Linkage to a PD susceptibility locus has been detected using four different studies of multiplex PD families. In the PROGENI study, initial linkage analyses performed in a sample of 90 families with 96 affected sibling pairs meeting the narrow PD definition produced a maximum lod score of $1.6 .^{4}$ Analyses in the GSPDEuropean Consortium consisting of 199 families yielded a maximum lod score of 1.05 near the marker D5S471 using 


\section{PROGENI Pankratz et al. 2003 GSPD — Martinez et al. 2004 \\ Li et al. $2002 \longrightarrow$ Scott et al. 2001

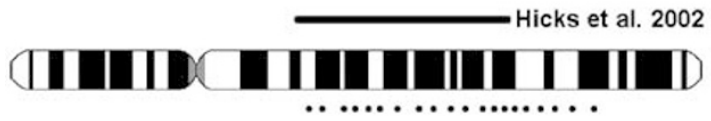

Figure 1 Evidence of linkage to chromosome $5 q$ from four studies. The location of the markers used for fine mapping in this study are represented as dots below the ideogram.

a narrow disease definition. The evidence of linkage was greatest (lod $=2.2$ ) among those 115 families that did not provide evidence of linkage to chromosome $2 .{ }^{5}$ A genome screen in a sample of 51 Icelandic families resulted in a maximum lod score of 1.6 on chromosome $5 \mathrm{q}$ near the marker D5S666. ${ }^{6}$ The Duke PD study, using 174 families generated a maximum lod score of 1.5 on chromosome $5 \mathrm{q}$ near the marker D5S816. ${ }^{7}$ Further studies in these families also detected linkage to a gene that may contribute to PD age of onset in this same region of chromosome $5 \mathrm{q} .{ }^{8}$ Clearly, the evidence from each study individually is relatively modest; however, the convergence of linkage evidence from so many different studies suggests that a gene with moderate effect on PD risk may be located in this region of chromosome 5 .

To test rigorously whether a gene contributing to $\mathrm{PD}$ susceptibility or PD age of onset is found on chromosome $5 \mathrm{q}$, a global genetics consortium was established between the PROGENI and GSPD-European Consortium studies. The goal of this consortium was to genotype a common set of markers across the region linked to PD susceptibility and test within an expanded sample of multiplex PD families whether a gene affecting the risk for PD was located on chromosome 5 .

\section{Methods}

\section{Consortium samples}

The PROGENI study samples were recruited through 59 Parkinson Study Group (PSG) sites located throughout North America. The sample included 832 affected individuals with DNA from 379 multiplex PD families ascertained through a sibling pair reportedly affected with PD. All study participants completed a uniform clinical evaluation (UPDRS; ${ }^{9}$ ) and a Diagnostic Checklist. ${ }^{4}$ The Diagnostic Checklist was developed with inclusion criteria consisting of clinical features highly associated with autopsyconfirmed PD and exclusion criteria highly associated with other non-PD pathological diagnoses. ${ }^{10,11}$ Responses on the Diagnostic Checklist were used to classify study subjects as having verified PD or nonverified PD. Age of onset information was available for 769 of the 832 sampled individuals. Twelve percent of those with age of onset information in the PROGENI sample had an age of onset of
45 years or younger, the typical criteria for early onset PD. Screening for mutations in known PD genes was not performed in all study samples. The majority of families (85\%) consisted of a single pair of affected siblings. The PROGENI sample was primarily Caucasian (94\%), although Hispanics (5\%) also participated. The male-to-female ratio in this sample was 1.2. Appropriate written informed consent approved by each individual institution's Institutional Review Board was completed.

The GSPD-European Consortium sample consisted of 654 genotyped individuals (406 affected) from 199 multiplex PD pedigrees, ascertained through an index case with a definite diagnosis of PD. One hundred and eightyeight pedigrees were ascertained from five European countries (France - 70, United Kingdom - 33, Netherlands -26 , Germany - 30 and Italy - 29), and the remaining 11 pedigrees were recruited in the United States. Following a standardized neurological evaluation, ${ }^{5}$ subjects were classified as definite PD if the following criteria were met: presence of three of the four cardinal signs of PD (bradykinesia, rigidity, rest tremor, asymmetry of signs at onset), at least 30\% improvement with levodopa therapy and absence of exclusion criteria (supranuclear gaze palsy, Babinski sign, cerebellar signs, dyspraxia, prominent and early urinary symptoms, MMSE $<24 / 30$ within 2 years of onset). Probable PD was defined as three of the four cardinal signs, or two of the four cardinal signs and at least $30 \%$ improvement with levodopa therapy, and absence of exclusion criteria. Age of onset information was available from 388 affected individuals. There was no difference in the subject's age at participation, age at onset or clinical symptoms between the six ascertainment sites. ${ }^{5}$ Thirteen percent of those with age of onset information in the GSPD-European Consortium sample had an age of onset of 45 years or younger, the typical criteria for early-onset PD. Screening for mutations in known PD genes was not performed in all study samples. The sample was nearly exclusively Caucasian with an affected male-to-female ratio of 1.1.

\section{Molecular genotyping and statistical analyses}

A common set of 20 equally spaced, microsatellite markers, with an average intermarker density of $4.3 \mathrm{cM}$, was genotyped across a $79 \mathrm{~cm}$ region of chromosome $5 \mathrm{q} 23$ region. The PROGENI and GSPD-European Consortium samples were genotyped in different laboratories using previously described methods. ${ }^{4,5}$ A CEPH control sample (1347-02) was genotyped in both laboratories to assist in combining the two samples for joint analyses.

A genome screen had been previously been completed in both the PROGENI and GSPD-European Consortium samples. Results from the screen had been used to verify all family structures. The data from the 20 markers genotyped as part of this study was evaluated for Mendelian inheritance of marker alleles using the program 
Pedcheck. ${ }^{12}$ The distribution of the marker allele sizes and frequencies were reviewed in each sample and were found not to be significantly different. To further test for subtle differences in allele frequencies within the GSPD-European Consortium sample, given the varied origin of the families, examination of the allele frequencies was performed based on the country of origin of the family. A $\chi^{2}$ test of homogeneity was performed comparing the marker allele frequency distribution for samples ascertained across the six GSPD-European Consortium sites. Only marker alleles with a frequency of $5 \%$ or greater were included in the analyses. The PROGENI study does not provide sufficiently detailed ethic information to further stratify families of Caucasian, non-Hispanic descent. Therefore, allele frequencies in the PROGENI sample were compared between the Caucasian Hispanic and non-Hispanic families. We did not detect significant differences in allele frequencies in any of these comparisons.

For each marker, two approaches were employed to combine the genotypic data for a joint analysis. In the first method, the allele sizes and frequencies were compared along with the control sample genotypes to align the marker alleles in the two samples. The frequency of the aligned markers were computed in the joint samples and employed for all analyses. Rare alleles were binned into the nearest allele that the two studies had in common. In the second method, the loci are duplicated, one for each data set, and analyses were performed using samplespecific allele frequencies. There were minimal differences between the two methods, so results are only presented using the marker alignment method.

Model-free tests of linkage (Merlin; ${ }^{13}$ ) were employed for statistical analyses. Allele frequencies were estimated using all alleles genotyped in the two studies. The extent of allele sharing among affected relative pairs was used to test for a PD susceptibility gene on chromosome 5q23. Two disease models, one narrower and one broader, were employed for genetic analyses (see Table 1). To employ genotypic information from all available family members, those genotyped family members which were not considered affected within a particular model were coded as unknown. A second series of analyses was also performed to assess the evidence of linkage for a gene contributing to the age of onset of PD to the chromosome $5 \mathrm{q} 23$ region. A variance component approach as implemented in Merlin was employed. Analyses were performed to test for linkage with age of PD onset using only the samples and families meeting criteria for the narrower PD definition and subsequently broadening the sample to include those individuals and families meeting criteria for the broader PD definition. One thousand replicates of the combined data were generated under the null hypothesis of no linkage using the program Merlin to estimate the probability of the observed maximal lod score.

\section{Results}

Evidence of linkage to a PD susceptibility gene using either the narrower or broader definition of disease was minimal in the combined data set (Figure 2). The maximum lod score $(\operatorname{lod}=0.2)$ using the narrower disease definition was in the midpoint of the region, near the marker D5S2011. Using the broader disease model, the maximum lod score of 0.4 was obtained at the marker D5S2049, $20 \mathrm{cM}$ distal to the maximum obtained with the narrower model.

To better interpret these results, the analysis was also performed in the two consortium samples separately. The PROGENI families had little evidence of linkage across the entire $70 \mathrm{~cm}$ interval. The highest lod score using either model of affection was obtained using the broader disease definition near the marker D5S2055 (lod=0.6). The GSPDEuropean Consortium families provided greater evidence of linkage with a maximum lod score of 1.3 obtained using

Table 1 PROGENI and GSPD-European Consortium samples

\begin{tabular}{|c|c|c|c|}
\hline & $\begin{array}{l}\text { PROGENI study } \\
\text { Verified PD }\end{array}$ & $\begin{array}{c}\text { GSPD-European Consortium sample } \\
\text { Definite PD }\end{array}$ & Combined sample \\
\hline \multicolumn{4}{|l|}{ Narrower model } \\
\hline No. of affected individuals & 435 & 376 & 811 \\
\hline No. of unaffected individuals & 39 & 246 & 285 \\
\hline No. of families & 208 & 179 & 387 \\
\hline No. of affected sibling pairs & 234 & 193 & 427 \\
\hline \multirow[t]{2}{*}{ Age of onset, years (mean \pm s.d.; range) } & $60.7 \pm 11.6(22-84)$ & $57.8 \pm 11.0(24-84)$ & $59.5 \pm 11.5(22-84)$ \\
\hline & Verified and nonverified PD & Definite and probable PD & \\
\hline \multicolumn{4}{|l|}{ Broader model } \\
\hline No. of affected individuals & 832 & 406 & 1238 \\
\hline No. of unaffected individuals & 17 & 248 & 265 \\
\hline No. of families & 379 & 190 & 569 \\
\hline No. of affected sibling pairs & 470 & 216 & 686 \\
\hline Age of onset, years (mean \pm s.d.; range) & $61.1 \pm 12.1(18-87)$ & $57.9 \pm 11.0(24-84)$ & $60.0 \pm 11.9(18-87)$ \\
\hline
\end{tabular}




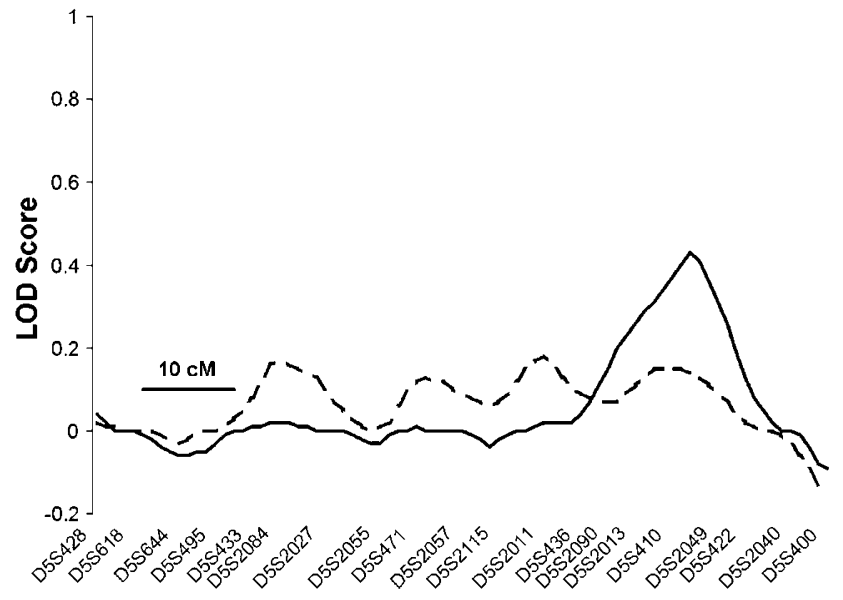

Figure 2 Evidence of linkage to chromosome $5 q$ using two models of PD susceptibility. The solid line depicts results using the broader disease definition and the dashed line depicts results with the narrower disease definition.

the narrower disease definition at a marker $4 \mathrm{~cm}$ away (D5S471). When the broader model was employed, results were very similar (lod $=1.0$ at marker D5S471). Review of the information content in the two samples suggests that the PROGENI sample consisting primarily of single pairs of affected siblings is less informative. The average information content in these families was 54\%. In contrast, the GSPD-European Consortium families, which more frequently contain unaffected genotyped individuals and often consist of more than two affected siblings, had a higher average information content of $65 \%$.

Analyses were also performed to test the hypothesis that a gene contributing to the age of onset of PD may be located within the chromosome 5q23 region (Figure 3). Analyses in the combined set of families provided evidence of linkage to the quantitative age of onset phenotype in the most distal region of the tested interval, at the marker D5S2040, with a maximum lod score of 1.8 when the broader definition of PD was employed. In addition, the most proximal portion of the tested interval also provided some evidence of linkage with a maximum lod score of 1.6 near the marker D5S428. The narrower disease definition produced lod scores below 0.5 throughout the $5 \mathrm{q} 23$ region.

In contrast to the results with the PD susceptibility gene analyses, the greatest evidence of linkage to the quantitative age of onset phenotype was observed in the PROGENI samples when the broader disease model was employed. A maximum lod score of 2.0 was obtained near the marker D5S422 in the more distal region of chromosome $5 \mathrm{q}$, corresponding to an empirical $P$-value of 0.065 . A second linkage peak (lod $=1.5)$ was seen in the more proximal region near the marker D5S428. Evidence of linkage to a gene contributing to the variation in age of onset was minimal in the smaller set of PROGENI families meeting the narrower disease definition $(\operatorname{lod}<0.25)$. The GSPD-

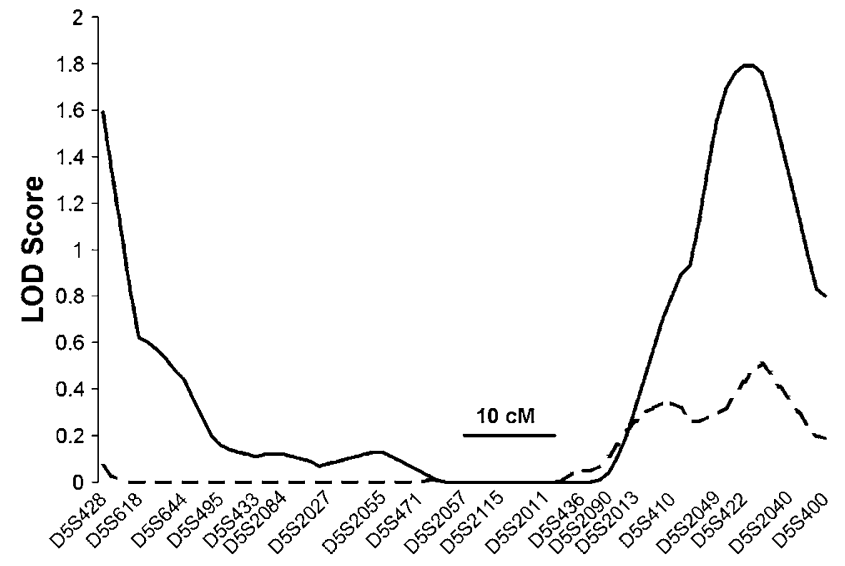

Figure 3 Evidence of linkage to chromosome $5 q$ using age of onset of PD as the phenotype. Analyses limited to only those individuals meeting the criteria for the narrower definition of disease are depicted with the dashed line and results with the expanded sample meeting the criteria for the broader disease definition are shown with the solid line.

European Consortium families produced lod scores less than 0.75 throughout the chromosome $5 \mathrm{q} 23$ region when analyzing the age of onset phenotype.

\section{Discussion}

This study was designed to evaluate rigorously the evidence for a gene on chromosome 5 contributing to PD susceptibility in the largest sample of familial PD ever analyzed using linkage methods. We have analyzed a sample of 1238 affected individuals from 569 multiplex ascertained throughout North America and Europe. The subjects were rigorously evaluated and a common set of microsatellite markers was genotyped at approximately $5 \mathrm{~cm}$ intervals throughout a $79 \mathrm{~cm}$ region.

Previously, evidence of linkage to chromosome 5 had been obtained from four independent samples, although the linkage results in each sample was modest. Despite the large sample and extensive marker genotyping performed in the current study, we did not detect significant evidence of linkage to a PD susceptibility gene on chromosome 5 . To ensure maximal power to detect linkage, analyses were performed using two disease models. There was greater evidence of linkage in the GSPD-European Consortium sample than in the PROGENI sample. However, even the evidence in the primarily European sample did not reach statistical significance. It should be noted that the sample of PD families is relatively heterogeneous. Although the sample is primarily Caucasian in origin, the families in this study were recruited from seven different countries. As a result, it is possible that our ability to detect linkage may be reduced owing to underlying genetic heterogeneity. This is particularly likely if a PD susceptibility gene on 
chromosome 5 was only segregating in a subset of families. With this potential limitation, we interpret our results in a large sample to suggest that if there is a PD susceptibility gene on chromosome 5 , it is unlikely to have major or even moderate effect on disease risk.

Owing to the evidence of linkage to chromosome $5 \mathrm{q}$ using the age of PD onset as a quantitative phenotype, ${ }^{8}$ we performed additional analyses to evaluate if the previously reported linkage results might be owing to a gene, which affected when an individual develops PD rather than their disease susceptibility. Higher lod scores were obtained when analyzing age of onset of PD. Similar evidence of linkage was observed at both ends of the genotyped region. However, even these linkage results are modest and do not meet the criteria for statistically significant linkage evidence. Interestingly, the evidence for linkage to a gene contributing to the variation in PD age of onset is greater in the PROGENI sample than in the GSPD-European Consortium sample, the reverse of the findings observed when analyzing PD susceptibility. These differing results using age of onset as phenotype may be owing to the greater size of the PROGENI sample and its corresponding greater power to detect linkage to a quantitative trait. However, it must be stressed that none of our linkage findings were statistically significant.

Evaluation of several candidate genes in the chromosome $5 q$ region, including the alpha-synuclein-interacting protein gene (SNCAIP, also known as synphilin-1), has not resulted in the identification of any significant disease associations, ${ }^{14-16}$ although a potentially putative mutation was reported. ${ }^{17}$ Sequencing of the SNCAIP gene in the PROGENI samples did not identify any mutations (unpublished data). These results in combination with the analyses performed in the current study strongly suggest that it is unlikely that there is a gene of major or moderate effect on chromosome $5 q$ contributing to PD susceptibility or PD age of onset in familial PD.

\section{Acknowledgements}

This project was supported by NS37167 (to TF) and the Michael J Fox Foundation for Parkinson's Research, Edmond J Safra Global Genetics Consortia (to TF, MM and NW). This work was also supported by grants from the European Community Biomed 2 (BMHCT960664), National Institutes of Health (Grant NS41723-01A1 to AB), the Parkinson's disease society and the Brain Research Trust (to NW), BMBF (Grant No. 01 GI 0201, Kompetenznetzwerk Parkinson to TG), the National Network for Genome Research (Grant No. 01 GSO116 to TG), 'Ministero dell'Istruzione, Universita' e Ricerca', MIUR, Italy (to $G M$ and VB), the NINDS Udall Center (to JH and MF), the NIA intramural program (JH) and NIH/NINDS P01 NS40256 (MF). We thank the members of the French Parkinson's Disease Genetic Study Group for contributing to the recruitment of families.

\section{References}

1 de Rijk MC, Tzourio C, Breteler MM et al: Prevalence of parkinsonism and Parkinson's disease in Europe: the EUROPAR-
KINSON Collaborative study. European community concerted action on the epidemiology of Parkinson's disease. I Neurol Neurosurg Psychiatry 1997; 62: 10-15.

2 Gasser T: Genetics of Parkinson's disease. J Neurol 2001; 248: 833-840.

3 Gasser T: Genetics of Parkinson's disease. Curr Opin Neurol 2005; 18: $363-369$.

4 Pankratz N, Nichols WC, Uniacke SK et al: Genome screen to identify susceptibility genes for Parkinson disease in a sample without parkin mutations. Am J Hum Genet 2002; 71: 124-135.

5 Martinez M, Brice A, Vaughan JR et al: Genome-wide scan linkage analysis for Parkinson's disease: the European genetic study of Parkinson's disease. J Med Genet 2004; 41: 900-907.

6 Hicks AA, Petursson H, Jonsson T et al: A susceptibility gene for late-onset idiopathic Parkinson's disease. Ann Neurol 2002; 52: 549-555.

7 Scott WK, Nance MA, Watts RL et al: Complete genomic screen in Parkinson disease: evidence for multiple genes. JAMA 2001; 286: 2239-2244.

$8 \mathrm{Li} \mathrm{YJ}$, Scott WK, Hedges DJ et al: Age at onset in two common neurodegenerative diseases is genetically controlled. Am J Hum Genet 2002; 70: $985-993$.

9 Lang AE, Fahn S: Assessment of Parkinson's disease; in Munsat TL (ed): Quantification of neurologic deficit. Boston: Butterworth, 1989, pp 285-309.

10 Hughes AJ, Daniel SE, Kilford L, Lees AJ: Accuracy of clinical diagnosis of idiopathic Parkinson's disease: a clinico-pathological study of 100 cases. J Neurol Neurosurg Psychiatry 1992; 55: $181-184$.

11 Hughes AJ, Ben Shlomo Y, Daniel SE, Lees AJ: What features improve the accuracy of clinical diagnosis in Parkinson's disease: a clinicopathologic study. Neurology 1992; 42: 1142-1146.

12 O'Connell JR, Weeks DE: PedCheck: a program for identification of genotype incompatibilities in linkage analysis. Am J Hum Genet 1998; 63: 259-266.

13 Abecasis GR, Cherny SS, Cookson WO, Cardon LR: Merlin - rapid analysis of dense genetic maps using sparse gene flow trees. Nat Genet 2002; 30: 97-101.

14 Maraganore DM, Farrer MJ, Lesnick TG et al: Case-control study of the alpha-synuclein interacting protein gene and Parkinson's disease. Mov Disord 2003; 18: 1233-1239.

15 Bandopadhyay R, de Silva R, Khan $\mathrm{N}$ et al: No pathogenic mutations in the synphilin-1 gene in Parkinson's disease. Neurosci Lett 2001; 307: 125-127.

16 Satoh JI, Kuroda Y: A putative polymorphic Val44Ala variation in the synphilin-1 gene is undetectable in Japanese sporadic Parkinson's disease patients. Eur J Neurol 2002; 9: 15-18.

17 Marx FP, Holzmann C, Strauss KM et al: Identification and functional characterization of a novel R621C mutation in the synphilin-1 gene in Parkinson's disease. Hum Mol Genet 2003; 12: $1223-1231$.

\section{Appendix A}

For PROGENI: William C Nichols (Cincinnati Children's Hospital Research Center, USA), Parkinson Study GroupPROGENI Investigators. For GSPD-European Consortium: Christine Betard (CNG, France), Vincenzo Bonifati, Giuseppe Meco (University 'La Sapienza', Rome, Italy), Alexis Brice, Alexandra Durr (INSERM U679, France), Thomas Gasser (University of Tubingen, Germany), Giuseppe De Michele (Federico II University, Naples, Italy), Ben Oostra (Erasmus MC, Rotterdam, Netherlands), Nicholas W Wood (Institute of Neurology, UK), Matthew Farrer (Mayo Clinic, Jacksonville, USA), John Hardy (National Institute on Aging, USA). 